\title{
Interoperability: a reliability challenge across systems and humans; illustrated by health care examples
}

\author{
Gerrit Muller \\ Buskerud University College and Embedded Systems Institute \\ Gerrit.muller@embeddedsystems.nl \\ Copyright $@ 2008$ by Gerrit Muller. Published and used by INCOSE with permission.
}

\begin{abstract}
End-to-end functionality is often the result of cooperation between many systems. We will discuss interoperability of systems, including the humans involved. Root causes of interoperability problems are inconsistencies between the models of the world within the system and the actual situation in the world. The dynamics of time complicates interoperability even more.

Standards play a crucial role in facilitating interoperability. Unfortunately, compliance to standards is no guarantee for interoperability. Interoperability is required at many levels, from single technical systems to social systems. Abstraction is one of the means to cope with increased interoperability scope. However, rather detailed implementation issues may cause dangerous interoperability problems.

Interoperability has many dimensions: technical, applicational, social, and cultural. The ownership for interoperability is unclear. This unclear ownership in such multi-dimensional problem space explains why interoperability is so difficult to achieve.
\end{abstract}

\section{Introduction}

A lot of functionality nowadays requires complex interaction between multiple systems and humans. The degree in which systems interoperate successfully is called interoperability. We may view the involved systems and humans as a system-of-systems [Maier 1998], where individual systems are autonomous and where the integral functionality and performance emerges. The actual end-to-end use of the interoperating systems is not a priori designed, but instead it evolves over time.

In this paper we will show some safety and reliability problems that were caused by imperfect interoperability. As an example we take an incident that took place in Dominican Republic where the appendix of the wrong person has been removed due to limited interoperability between systems. We will also discuss some more examples from the health care domain.

Interoperability plays a role in many domains: defense, health care, traffic control, entertainment, telecommunication, finance and administration, manufacturing, et cetera. The first step towards interoperability is the definition of and the compliance with standards. We will discuss the role of standards in achieving interoperability.

Any mismatch in connectivity or interoperability shows up first as functionality or performance problem and then as intermittent reliability (or safety, security, availability) problem. Imperfect interoperability between systems and humans is a major threat to the dependability on end-to-end functionality in many domains.

Interoperability seems to be a poorly understood characteristic that in the authors experience is often approached with great naivety. For example, large programs where compliance to 
standards is mistaken to be the same as interoperability of systems. In this paper we will discuss the relation between individual systems, the involved humans, and the overall resulting interoperability.

\section{Analysis of health care interoperability problem}

The Santa Cruz Sentinel published an article at May 23, 2008 containing this text:

“... The mistake occurred Nov. 14 when two female patients were scheduled for computed tomography, or CT scans, according to the state report. The first patient underwent an appendectomy that very evening because of the CT results. But the surgery was unnecessary. The next day, a radiologist discovered the patient's CT scan was actually that of a second patient.

Hospital staff told state inspectors that the technologist had trouble starting the required intravenous line for the first patient and took her out of the CT scan room to complete that task.

However, the patient's information had already been entered into the computer system for the CT scan. After the second patient's scan was completed, a radiology technician noted the error, removed the first patient's information and entered information on the second patient.

When the first patient's information was deleted from the computer in the scan room, it was not deleted from the computer system used by the radiologist.

"This was due to an incompatibility of the software between the two systems," the state report said. ..."

Figure 1 visualizes the events in time, relating human activities to the information stored in the involved systems. Core of the problem is that first the image data of the second patient is labeled with the first patient's name, while later the correction of this name is not propagated to other systems that received copies of the same data.

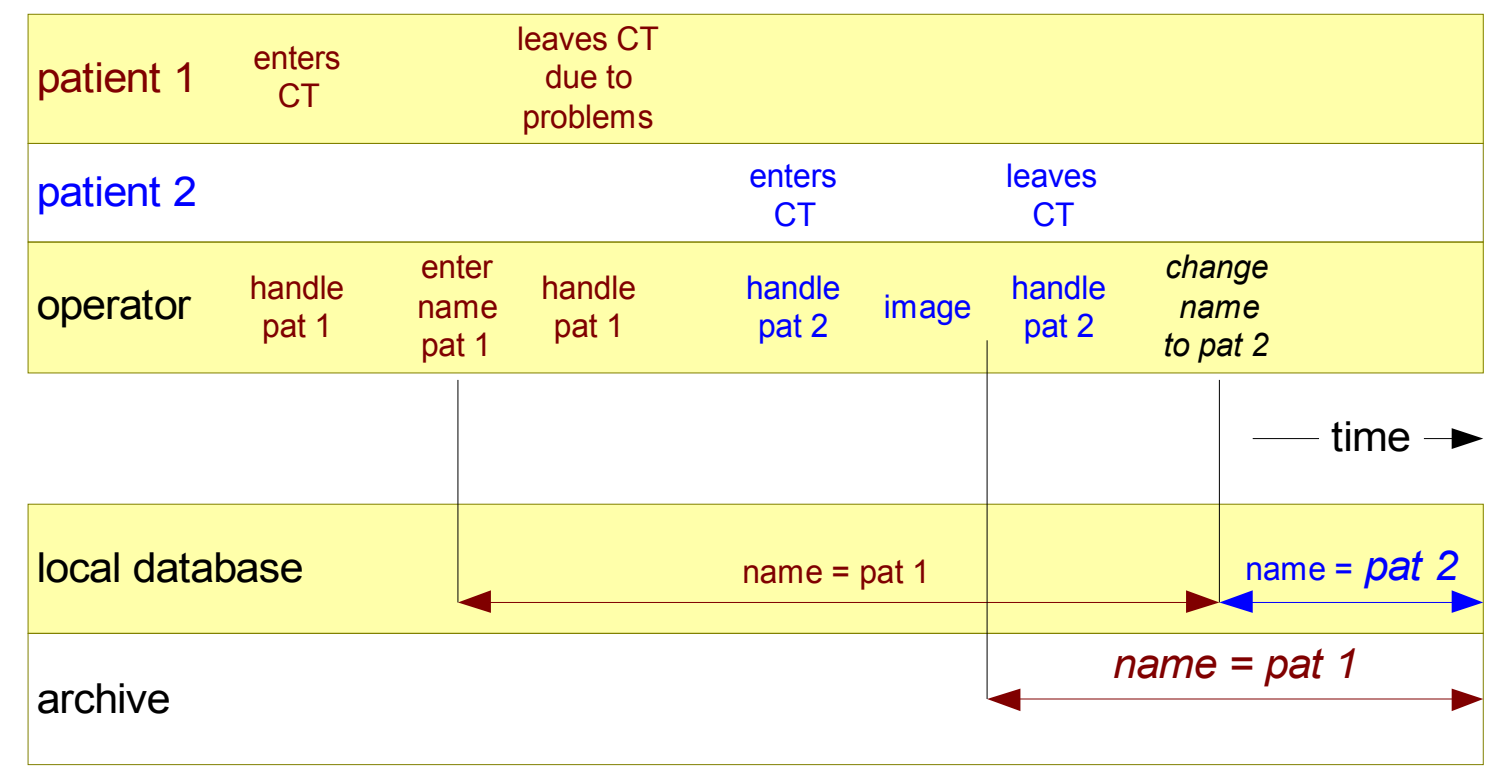

Figure 1, Visualization of the appendix removal case, showing human activities in relation to the information within the involved systems.

Figure 2 broadens the scope of the systems and the stakeholders to cover the chain of events from admission to the hospital, where the patient information is entered via a standard Personal Computer (PC) in the Radiology Information System (RIS), up to the surgery where the wrong 
person has been operated. Note that we deliberately simplified this diagram. Nevertheless we can already see the complexity that arises from the number of involved systems and human stakeholders.

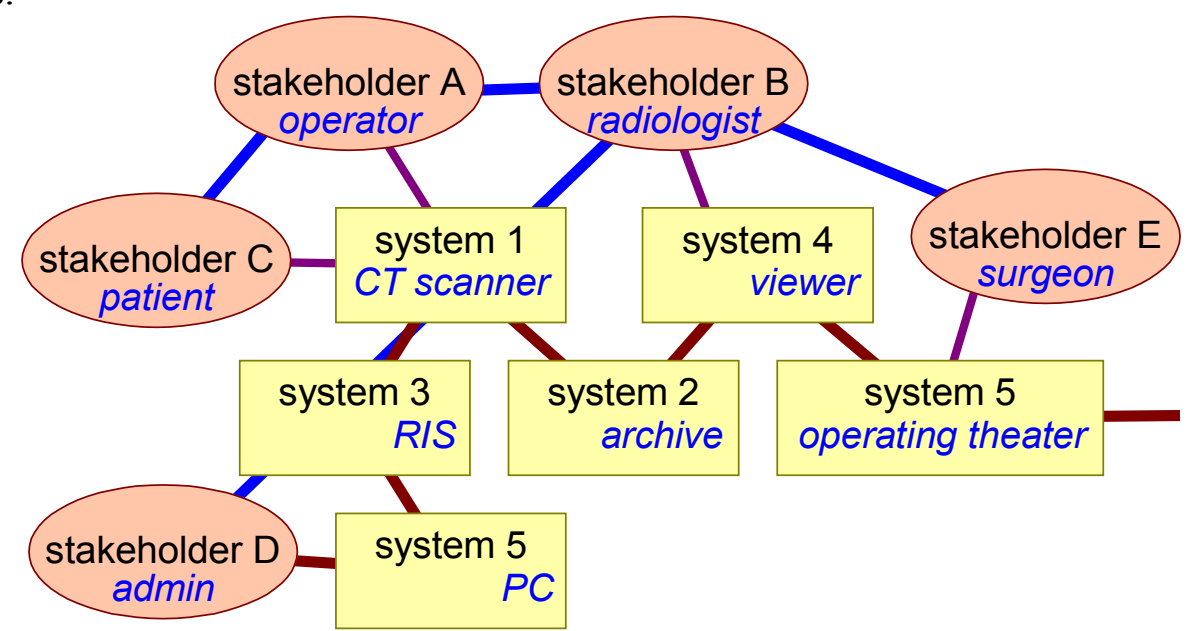

Figure 2, Involved stakeholders and systems seen from a broader perspective.

The interoperability between health care systems is facilitated by several standards, such as HL7 defining patient and care related information model, DICOM defining image related information model and HIPAA regulating health care related information use. Figure 3 shows these standards as the starting point for the specifications of the involved systems. These specifications are transformed by development engineers into a design. The design is manufactured many times to create actual physical instances of the systems to be used in hospitals.

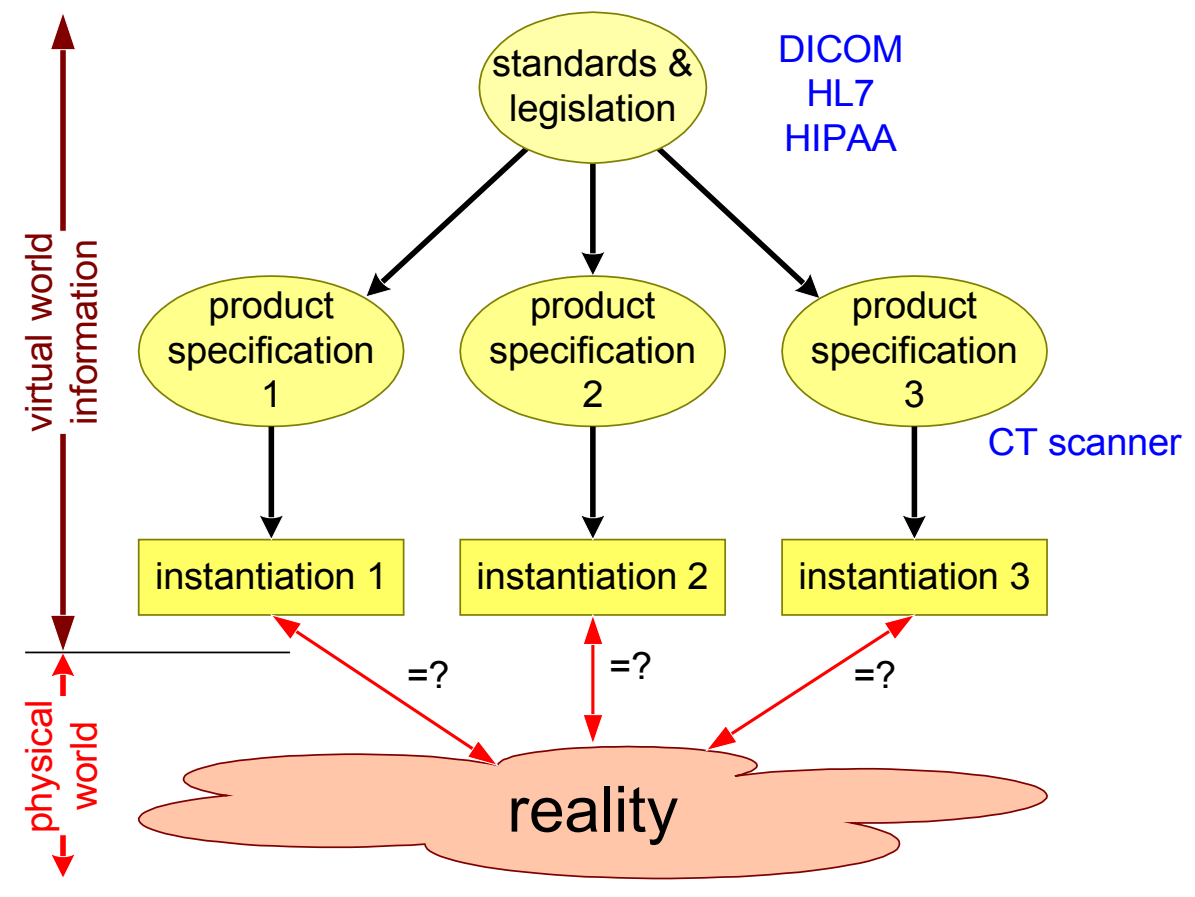

Figure 3, Distance between definition in standards and the actual reality in the physical world. 
In every physical system software is running that maintains a local view on the external physical world. Figure 1 showed that the local database of the CT scanner "thinks" that the first patient is lying in the system, while in the physical reality the second patient is in the system. This misinformation is copied into the archive system, causing more inconsistencies between the virtual views within the systems and the actual physical reality

Observation. Inconsistency between virtual views and physical reality is a major cause of dependability problems.

\section{Adding the time dimension}

Figure 3 is simplified by ignoring the time dimension. However, in all layers of the figure time plays a role. In the development of systems we see an evolution of standards, which is followed by the adaptation of existing systems and the creation of new systems according to the evolved standards. Here typical time constants are months to years. The instances of the systems and processes in the physical world have typical time constants from seconds to hours. The consistency between internal views of the systems and the actual situation is a dynamic challenge. What might be consistent now doesn't guarantee consistency in the near future. In the example of the removed appendix we see that one system gets consistent again after operator intervention, creating an inconsistency between CT scanner and archive.

In [Hendriks 2008] a more extensive model is provided showing the potential inconsistencies between internal views within systems and the actual physical situation. For traffic information systems this paper shows the impact of the dynamics on the interoperability of information gathering, processing and distribution.

\section{Standards and interoperability}

Figure 3 showed that standards form the starting point to create interoperability. Systems can only interoperate when these systems share a common view on the world. This common view is created by the standards. The standards build the common view in layers, as shown in Figure 4.

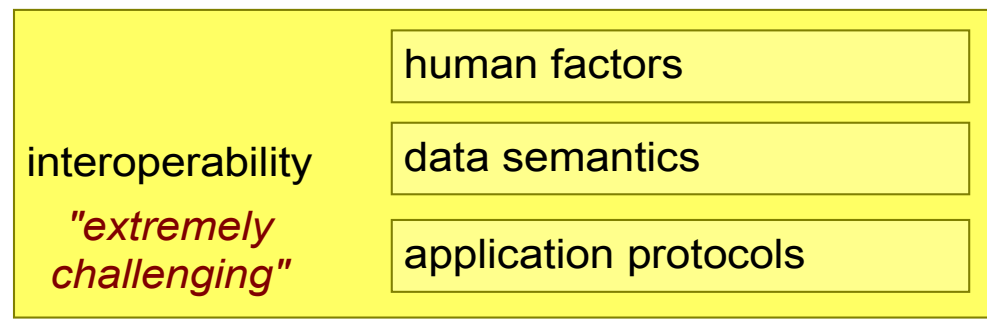

\begin{tabular}{|c|c|c|}
\hline \multirow{3}{*}{$\begin{array}{c}\text { connectivity } \\
\text { "only } \\
\text { engineering" }\end{array}$} & data syntax & $\begin{array}{r}\text { formats, } \\
\text { tags }\end{array}$ \\
\hline & protocols & e.g. TCP/IP \\
\hline & physical cab & $\begin{array}{l}\text { a, interfaces } \\
\text { nnectors, ... }\end{array}$ \\
\hline
\end{tabular}

Figure 4, layering of standards to create a common view required for interoperability. 
The more technical layers facilitate connectivity; the exchange of structured data between systems. Ensuring connectivity between systems is a matter of rigorous engineering. Much more challenging are the layers that address the applicational needs for interoperability. Here the technical world has to interface with the human world. The application protocols and data semantics are formalizations of processes and procedures in the domain. [Hendriks 2007] analyzes multiple implementations of the same navigation standard and shows all differences in interpretation and the severity for interoperability of navigation systems. Finally human factors play a significant role, since end-to-end interoperability involves many physical systems as well as many human stakeholders, see also Figure 2. [Boardman 2006] shows multiple scopes op systems of systems with increasing human factors in the defense domain.

\section{Compliance testing and interoperability}

Creating and building interoperable systems requires compliance testing of the applied standards. Unfortunately, compliance with these standards does not at all imply interoperability. [Moseley 2004] provides a description of compliance testing and interoperability testing. In this paper it is asserted that neither tests will guarantee interoperability, although the combination is described as complementary. Major challenge of interoperability testing is the open-endedness of the concept of interoperability, limiting the rigor of these tests.

Note that testing gets more difficult and the rigor of testing gets less when we go up in the layers shown in Figure 4. Connectivity in general is well matured and can be achieved by engineering and testing. Interoperability builds on connectivity, but is much more challenging to achieve.

\section{Broader scopes of interoperability in health care}

We have started by analyzing one local interoperability problem, the appendix case. However, the scope of health care systems is much broader. Stakeholders, both clinical as well as the patients, expect much more interoperability between all clinical processes and systems. Figure 5 shows a hierarchy of scopes where interoperability is expected: within systems, e.g. MR or CT scanners, within the department, e.g. radiology, within the hospital, or global. 


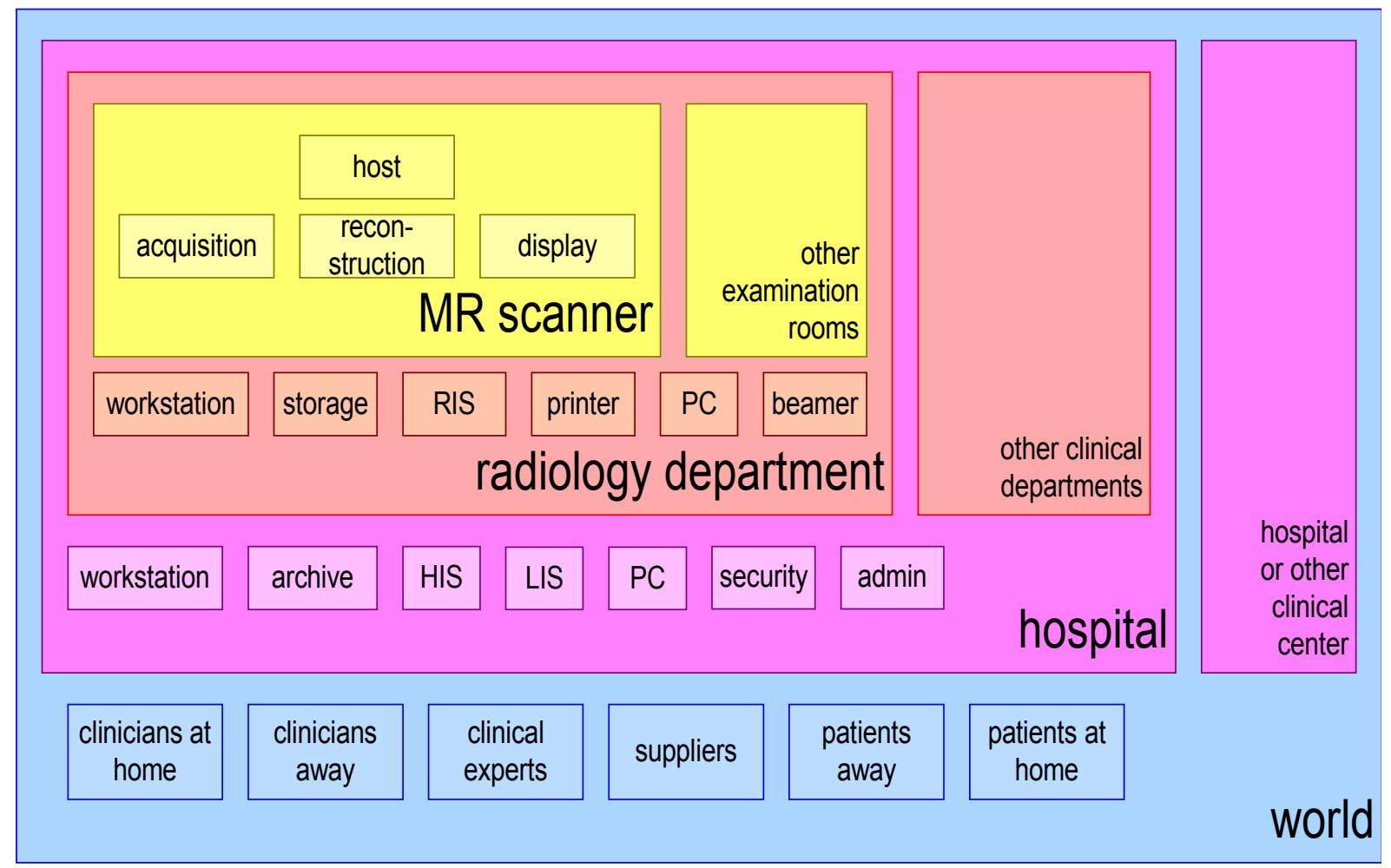

Figure 5, Hierarchy of health care scopes for interoperability.

In the clinical processes information is transformed and enriched while it flows from one operation to the next operation. Figure 6 shows a typical flow of information. In the graph it shows that the transformation of the data itself reduces the amount of data (e.g. selection of images, freezing of process steps). At the same time clinical information is added that enriches the clinical value of the information (e.g. annotation, addition of diagnosis).

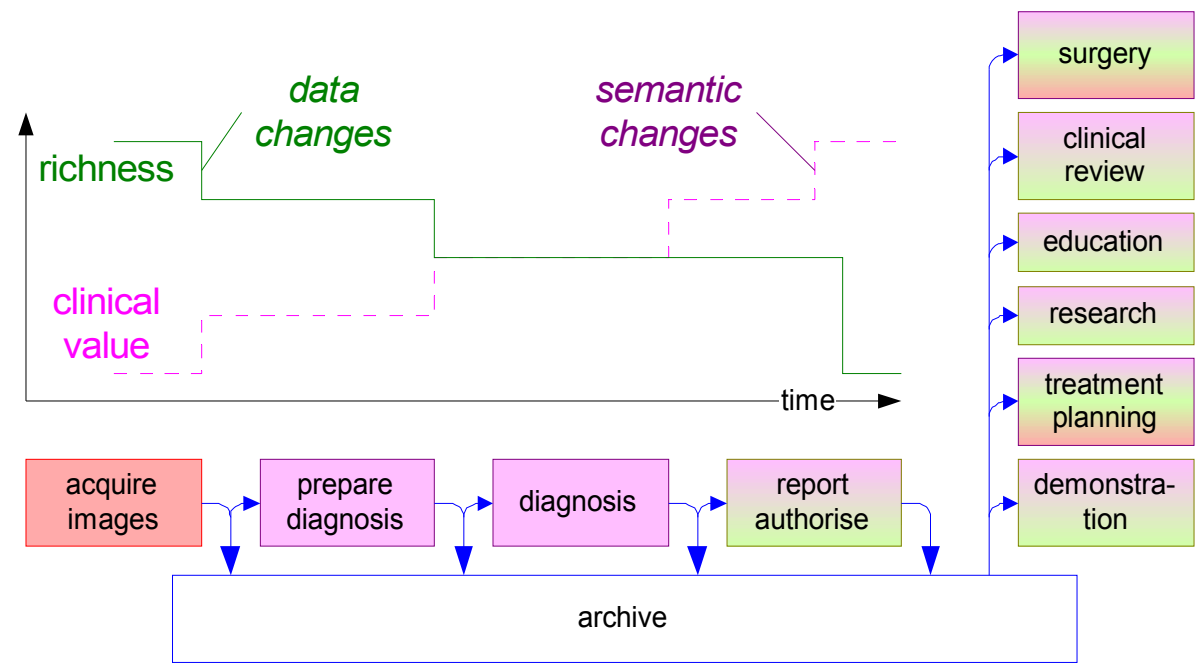

Figure 6, typical flow of information in a clinical process. 
This transformation of clinical information shows once more additional complexity caused by the time dimension. The format and the meaning of data depends on many contextual parameters: when, where, who, what, how. Figure 7 shows the multi-dimensionality of interoperability in a different way.

\begin{tabular}{|c|c|c|}
\hline integrating multiple & in multiple & delivered by multiple \\
\hline $\begin{array}{l}\text { applications } \\
\text { cilinical analysis } \\
\text { clinical support } \\
\text { administrative } \\
\text { financial } \\
\text { workflow }\end{array}$ & $\begin{array}{l}\text { languages } \\
\text { cultures } \\
\text { USA, UK, } \\
\text { China, India, } \\
\text { Japan, Korea } \\
\text { France, Germany } \\
\text { Italy, Mexico }\end{array}$ & $\begin{array}{c}\text { vendors } \\
\text { Philips } \\
\text { GE } \\
\text { Siemens }\end{array}$ \\
\hline based on multiple & and multiple & and multiple \\
\hline $\begin{array}{c}\text { media, networks } \\
\text { DVD+RW } \\
\text { memory stick } \\
\text { memory cards } \\
\text { bluetooth } \\
11 \mathrm{a} / \mathrm{b} / \mathrm{g} \\
\text { UTMS }\end{array}$ & $\begin{array}{c}\text { standards } \\
\text { Dicom } \\
\text { HL7 } \\
\text { XML }\end{array}$ & $\begin{array}{c}\text { releases } \\
\text { R5 } \\
\text { R6.2 } \\
\text { R7.1 }\end{array}$ \\
\hline
\end{tabular}

Figure 7, interoperability dimensions.

Different applications tend to have different views on the surrounding world. The information models that are the foundation for an application are based on the specific needs of that application. Integrating different applications requires mutual adaptations to create common views on exchanged information. Geographical differences add to the complexity in many ways: different social structures, legislation, language and culture. How to identify a Dutch patient in a Norwegian hospital? In the deployment of the systems many more variations pop up: multiple vendors, systems with different releases, different media and networks. Another complicating factor is that within one domain often multiple standards are required to get sufficient coverage of applications and functions.

\section{Evolution of standards.}

We have shown the applications, vendors and releases as dimensions of interoperability. More specific contexts allow for more specific interoperability. For example, within the catherization laboratory much higher degrees of interoperability can be achieved than in the broader context such as the hospital. These narrower niches of interoperability often require extensions to the generic standards that serve a much broader scope. However, in due time the additional information is also required in the broader context, such as the office of the cardiologist. In general standards evolve over time, where new functions and related information propagate from proprietary extensions to generic standards.

Figure 8 shows that this evolution may be gradual: from single vendor application specific to single vendor product family standard to single vendor standard to global standard. This kind of 
standardization processes take time, typical 5 years or more.

From interoperability point of view this evolution is a two-sided sword. It enables more intimate interoperability for the future, but at the same time it complicates interoperability of old and new systems. Nevertheless, interoperability expectations increase over time, the continuous evolvability of standards and systems is a fact.

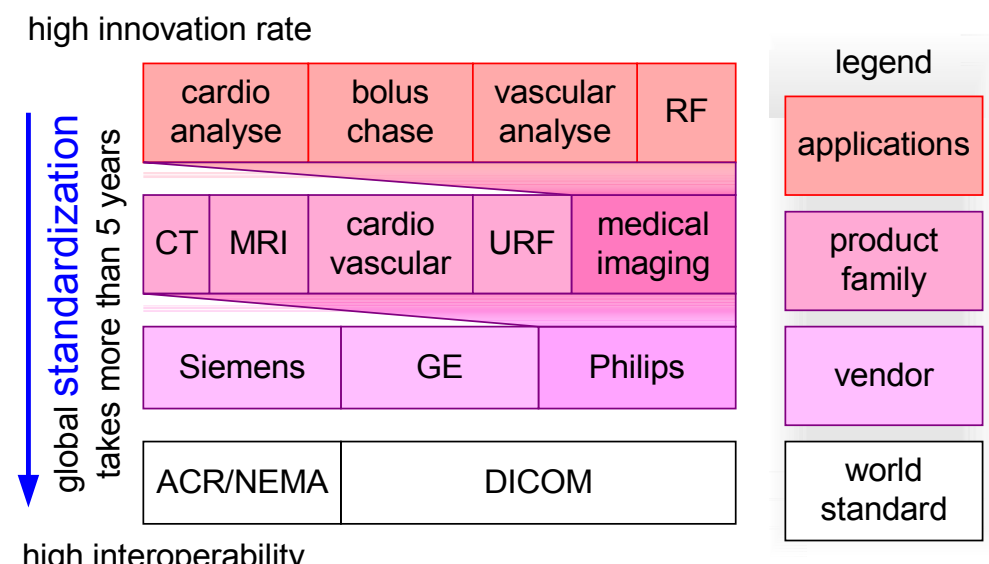

Figure 8, Evolution of standards to facilitate innovation.

\section{The devil is in the detail}

Interoperability problems can arise from rather detailed implementation and interpretation differences. Figure 9 shows an example of a detailed cause of interoperability: the position and size definition of annotations on an image. Images are acquired at an Universal Radiography Fluoroscopy (URF) system. The images can be annotated by the operator on this system, by means of a character overlay with fixed size letters. When the annotated images are exported to the workstation, then the workstation uses a slightly different rendering approach for the annotations, with different fonts and font sizes. An anchor point is used for positioning the annotation. The consequence is that the annotation may cover slightly different parts of the image on the two systems. The human procedure of using this systems is to use the " $>$ " symbol as arrow. The combination of different rendering implementations and the use of the symbol convention creates together a safety problem: the annotation might point to the wrong position on the workstation! 


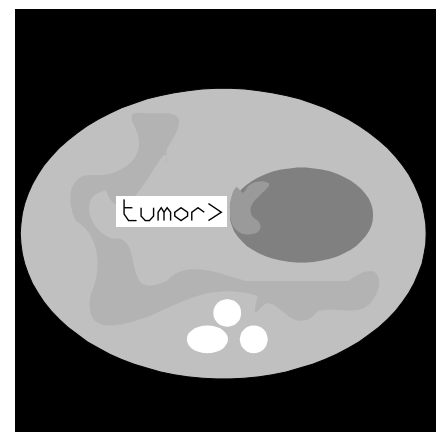

URF monitor output: fixed size letters at fixed grid

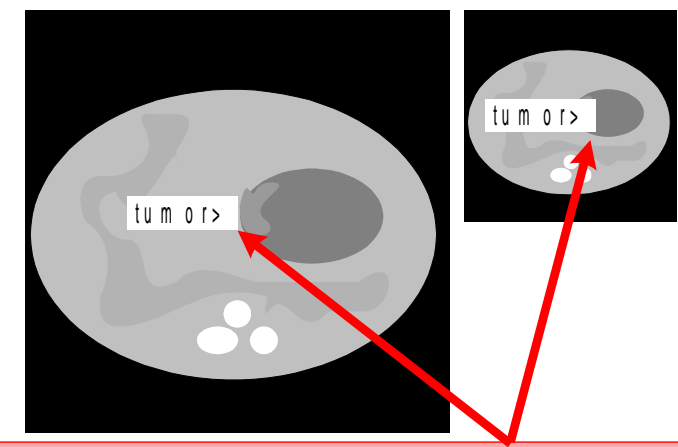

for user readability the font-size was determined "intelligently"; causing a dangerous mismatch between text and image

EV output: scaleable fonts in graphics overlay

Figure 9, example of an interoperability problem between an URF system and an EasyVision (EV) workstation.

\section{Who is responsible for interoperability?}

Interoperability problems are difficult to tackle due to the lack of ownership. Individual systems are defined, designed and verified by the creator of the system. Systems engineering is applied to achieve well behaving and well performing functions. The limits for single systems are already visible when the context is part of behavior and performance; What are the circumstances to test the system? What happens if the environment interferes with the system? At least for single systems the creator feels responsible for system performance.

When many systems and humans together form a system-of-systems the owner of the supersystem is often unclear. Who defines, designs and verifies behavior and performance of the super-system? Who provides feedback to individual systems to address interoperability issues? In practice solution providers start to appear who take somewhat this role. The challenge for this super-system designer and integrator is to cope with the total complexity and with the dynamics of the super-system. Many interoperability problems are caused by rather detailed domain issues between systems, as shown in Figure 9.

\section{Summary and Conclusion}

Interoperability is getting more and more important, since in many domains end-to-end functionality emerges from the cooperation of many systems and humans. Core to system of systems is the independence of the contributing systems. From interoperability point of view the lack of integral ownership is a problem. A solution provider who operates in the role of supersystem designer and integrator is very challenging: integrating independently created and evolving systems, operated by many humans, where low-level implementation details may cause dangerous interoperability problems. 


\section{References}

[Boardman 2006] J. Boardman et al. System of Systems. Characteristics and Interoperability in Joint Command and Control. Presentation at SoSECE 2006, Ft Belvoir VA, 2006

[Hendriks 2007] T. Hendriks and K. Wevers, Abstraction Conflicts in Industrial Deployment of Model-Based Interoperability Standards, In Proceedings: 5th Annual Conference on Systems Engineering Research, Hoboken, NJ, 14-16 March 2007.

[Hendriks 2008] T. Hendriks, The Impact of Independent Model Formation on Model-based Service Interoperability, 7th WSEAS Int. Conf. on ARTIFICIAL INTELLIGENCE, KNOWLEDGE ENGINEERING and DATA BASES (AIKED'08), University of Cambridge, UK, Feb. 2008.

[Maier 1998] Maier, M.W., "Architecting Principles for System of Systems," Systems Engineering, Vol. 1, No. 4, 1998, pp. 267-284.

[Moseley 2004] S. Moseley, S. Randall, A. Wiles, "In pursuit of interoperability", Journal of IT Standards \& Standardization Research, 2(2),34-48, July-Dec 2004

[Sanders 2008] Rex Sanders, Wrong patient gets appendix removed, software to blame, news item in comp.risks, May 2008, http://catless.ncl.ac.uk/Risks/25.17.html\#subj1

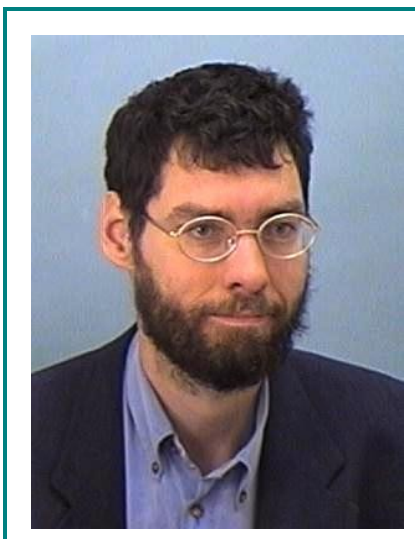

Gerrit Muller.

\section{Author Biography}

Gerrit Muller received his Master's degree in physics from the University of Amsterdam in 1979. He worked from 1980 until 1997 at Philips Medical Systems as a system architect, followed by two years at ASML as a manager of systems engineering, returning to Philips (Research) in 1999. Since 2003 he has worked as a senior research fellow at the Embedded Systems Institute in Eindhoven, focusing on developing system architecture methods and the education of new system architects, receiving his doctorate in 2004. In January 2008 he became a full professor of systems engineering at Buskerud University College in Kongsberg, Norway.

All information (System Architecture articles, course material, curriculum vitae) can be found at:

Gaudí systems architecting http://www.gaudisite.nl/ 